$$
\begin{aligned}
& \text { US -FT } 34-2 \\
& \text { Sw } 94.11
\end{aligned}
$$

US-FT $/ 2-94$

\title{
Inelastic diffractive production and string fusion in hadron-nucleus collisions
}

\author{
N. Armesto, M. A. Braun ${ }^{1}$ and C. Pajares \\ Departamento de Física de Partículas, \\ Universidade de Santiago de Compostela, \\ 15706-Santiago de Compostela, Spain
}

\begin{abstract}
The diffractive cross section in the string fusion model is evaluated for hadron-nucleus collisions. The resulting cross section is larger than in several models, including the Glauber model.
\end{abstract}

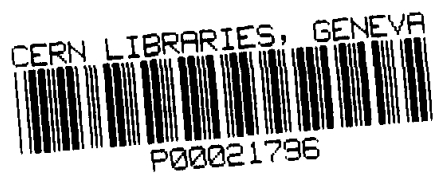

US-FT/2-94

February 1994

\footnotetext{
${ }^{1}$ On leave of absence from the Department of High Energy Physics, University of St. Petersburg, 198904 St. Petersburg, Russia.
} 


\section{Introduction}

The success of some semiphenomenological models for soft hadron interactions, such as the Dual Parton Model (DPM, [1], [2]) or the Fritiof model ([3], [4]), seems to confirm the idea that soft processes are mediated by multiple exchanges of some localized objects, coloured strings. Actually, it is the old Pomeron exchange picture what is revitalized in these models. The Pomeron is made of two strings. The number of exchanged Pomerons or pairs of strings is rather small for hadron-hadron collisions at present experiments but it rises with energy. Thus, the effect of interaction of strings may become important at extremely high energies or for central heavy nucleus collisions at not so high energies. This interaction may give rise to the fusion of strings, resulting in new strings with more colour charge ([5], [6]). These new objects could produce interesting effects ([7], [8]), specially the $\bar{\Lambda}$ enhancement and other antistrange particle enhancements observed.

The string fusion model was applied to study inelastic diffraction in hadron-hadron collisions ([9]). The diffraction receives contributions from two terms: the first one comes from the fluctuations in the number of interacting partons of the incoming hadron (once integrated over rapidity and impact parameter) and the second one from the diffractive part of the elementary amplitudes. In the string fusion model, the first contribution decreases with energy, therefore, to obtain a good agreement with the rising experimental data the second component is needed. This second component, as already said, depends on the diffractive part of the elementary amplitude characterized by the input ratio $\Omega^{0}=$ $\sigma_{0}^{i n} / \sigma_{0}^{i n+d}$. For $\Omega^{0}=0.79$ at $\sqrt{s}=23.5 \mathrm{GeV}$ and $\Omega^{0}=0.70$ at $\sqrt{s}=1800 \mathrm{GeV}$ we obtained $\sigma^{d}=6.02 \mathrm{mb}$ and $11.56 \mathrm{mb}$ respectively, in good agreement with the experimental data. A constant value of $\Omega^{0}=0.75$ results in $\sigma^{d}=7.03 \mathrm{mb}$ at $\sqrt{\mathrm{s}}=23.5 \mathrm{GeV}$ and $\sigma^{d}=9.46$ $m b$ at $\sqrt{s}=1800 \mathrm{GeV}$ in better agreement with the last Fermilab data.

In many multiple scattering models the diffractive part of the elementary amplitudes is neglected so that $\Omega^{0}=1$. The usual argument is that the elementary amplitude should have only short range correlations (like a one Pomeron exchange contribution). However. we think that there are neither experimental evidence nor theoretical foundations for this assumption. The existence of so-called enhanced Pomeron diagrams makes it evident that diffraction persists on the elementary level. Recall also Feynman's view on the similarity between the parton-parton amplitude and the hadron-hadron one ([10]).

In this paper we extend the analysis of inelastic diffractive production to hadronnucleus collisions. The total inelastic diffractive cross section obtained is larger than the corresponding to the Glauber model ([11], [12]) or the single diffractive cross section of the DPM ([13]). The predictions of these two models are also much larger than the existing data on the single diffractive dissociation of the nucleus $p A \rightarrow p X$, measured for $B e$, $A l$ and $W$ at the CERN Spp $S$ ([14]). The large values found in the string fusion model remain large even if the elementary amplitude is taken completely non-diffractive, $\Omega^{0}=1$. The increase of the inelastic diffractive cross sections is only of about 25 per cent for $\Omega^{0}$ going from 1 down to 0.7 .

The paper is organized as follows: In Section 2 the main formulae of the Glauber model are recalled in a slightly generalized approach to include inelastic diffraction in the 
elementary amplitude. In Section 3 the string fusion model is described and the formulae for the diffractive cross section are presented. In Section 4 the formulae of Sections 2 and 3 are compared. In Section 5 our results are presented and compared with several models. Finally, in Section 6 the conclusions are presented.

\section{Inelastic diffraction in the Glauber model}

For completeness, let us introduce the corresponding formulae for the inelastic diffractive cross section in the Glauber theory ([11], [12]). To describe the diffractive dissociation of the nucleus in a collision, one has to know its particle content, and not only of the nucleus itself but also of all its excited states, which are included in the diffractive spectrum. If one starts from a purely nucleonic picture of the nucleus, then of course all the nuclear states will be built of $A$ nucleons with different momenta, possibly bound in nuclear fragments of the same or lower $A$. However, this picture does not exactly correspond to the experimental situation, where the selection of diffractive events is made on the basis of their rapidity, rather than their nucleonic content. Therefore, we shall admit that the nucleus contains other components in addition to the simplest of $A$ nucleons. To stay within the Glauber picture we shall associate these additional components with an internal structure of each nucleon. In other words we assume that each nucleon in the nucleus may exist in a variety of states $|\alpha\rangle$. The precise nature of these states is irrelevant to us. In particular they can be made of many particles. The only requirement is that all of them do not include particles with rapidities greater than some threshold rapidity $y_{\max }$ in the lab system (Fig. 1). We use the same value $y_{\max }$ to define the elementary nucleon-nucleon target diffractive dissociation cross section $\sigma\left(y_{\max }\right)$ as the cross section for events with a spectrum of final particles having a rapidity gap starting in $y_{\max }$ up to the projectile rapidity $Y$.

The $S$-matrix is given by

$$
\begin{aligned}
S(b) & =\sum_{\alpha_{i}, \beta_{2}} \int\left[\prod_{i=1}^{A} d^{3} r_{i}\right] \Psi_{\alpha_{1}, \ldots, \alpha_{A}}^{*}\left(\vec{r}_{1}, \ldots, \vec{r}_{A}\right) \times \\
& \times \Psi_{\beta_{1}, \ldots, \beta_{A}}\left(\vec{r}_{1}, \ldots, \vec{r}_{A}\right)\left[\prod_{i=1}^{A} s_{\alpha_{i} \beta_{i}}\left(b-b_{i}\right)\right]
\end{aligned}
$$

where $\Psi_{\alpha_{1}, \ldots, \alpha_{A}}\left(\vec{r}_{1}, \ldots, \vec{r}_{A}\right)$ is the wave function of the nucleus, $\vec{r}_{i}=\left(\vec{b}_{i}, z_{i}\right)$ and $s_{\alpha \beta}(b)$ is the $S$-matrix for an individual collision at impact parameter $b$ of the projectile with a nucleon of the nucleus undergoing the transition from the state $|\beta\rangle$ to the state $|\alpha\rangle$.

As usual, we neglect correlations between the nucleons and assume that, first, the wave function factorizes into a spatial and an internal part and, second, the wave function factorizes in a product over nucleons,

$$
\Psi_{\alpha_{1}, \ldots, \alpha_{A}}\left(\vec{r}_{1}, \ldots, \vec{r}_{A}\right)=\prod_{i=1}^{A} \chi_{\alpha_{i}} \psi\left(\vec{r}_{i}\right)
$$


The integration over nucleonic coordinates is then done for each nucleon. Assuming that the range of the interaction is much smaller than the internucleon distances we obtain for each integral

$$
\int d^{3} r_{i}\left|\Psi\left(\vec{r}_{i}\right)\right|^{2} s_{\alpha \beta}=\delta_{\alpha \beta}+i a_{\alpha \beta} T(b),
$$

where $a_{\alpha \beta}$ is the forward projectile-nucleon scattering amplitude for the transition (of the nucleon) $|\beta\rangle \rightarrow|\alpha\rangle$ and $T(b)=\int d z|\Psi(\vec{b}, z)|^{2}$ is the usual profile function. Doing summations over $\alpha$ 's and $\beta$ 's we arrive at the standard Glauber formula

$$
S(b)=(1+i a T(b))^{A},
$$

with $a$ being the amplitude averaged over the excited nucleonic states inside the target nucleus,

$$
a=\sum_{\alpha, \beta} \chi_{\alpha}^{*} a_{\alpha \beta} \chi_{\beta}
$$

From Eq. (4) one inmediately obtains the formulae for the total, elastic and inelastic cross sections:

$$
\begin{aligned}
\sigma_{\text {tot }}^{h A}(b) & =2-(1+i a T(b))^{A}-\left(1-i a^{*} T(b)\right)^{A}, \\
\sigma_{e l}^{h A}(b) & =1-(1+i a T(b))^{A}-\left(1-i a^{*} T(b)\right)^{A}+|1+i a T(b)|^{2 A} \text { and } \\
\sigma_{i n+d}^{h A}(b) & =1-|1+i a T(b)|^{2 A} .
\end{aligned}
$$

To compute $\sigma_{e l+d(A)}^{h A}$ (the elastic plus nucleus diffractive dissociation cross section) we note that the $S$-matrix for the transition of the nucleus from its initial state $A$ determined by its wave function $\Psi$ to some other state $A^{\prime}$ with wave function $\Psi^{\prime}$ is given by the same Eq. (1) with the substitution of the final $\Psi$ for $\Psi^{\prime}$. Then the cross section is given by the squared modulus of the corresponding amplitude summed over $\Psi^{\prime}$. Using that the sum $\sum\left|\Psi^{\prime}\right\rangle\left\langle\Psi^{\prime}\right|$ acts as the unity operator in the subspace spanned by all the excited states of $A$ nucleons (this subspace does not cover all the Hilbert space which contains besides particle configurations with rapidities larger than $y_{\max }$ but less than $Y$ and so belonging neither to the nuclear nor to the projectile states) we get

$$
\begin{aligned}
\sigma_{e l+d(A)}^{h A}(b) & =\sum_{\alpha_{i}, \beta_{i}, \alpha_{i}^{\prime}} \int\left[\prod_{i=1}^{A} d^{3} r_{i}\right] \Psi_{\alpha_{1}, \ldots, \alpha_{A}}^{*}\left(\vec{r}_{1}, \ldots, \vec{r}_{A}\right) \Psi_{\alpha_{1}^{\prime}, \ldots, \alpha_{A}^{\prime}}\left(\vec{r}_{1}, \ldots, \vec{r}_{A}\right) \times \\
& \times\left(\prod_{i=1}^{A} s_{\alpha_{i} \beta_{i}}\left(b-b_{i}\right)-\prod_{i=1}^{A} \delta_{\alpha_{i} \beta_{i}}\right)\left(\prod_{i=1}^{A} s_{\alpha_{i}^{\prime} \beta_{i}}\left(b-b_{i}\right)-\prod_{i=1}^{A} \delta_{\alpha_{i}^{\prime} \beta_{i}}\right) .
\end{aligned}
$$

The terms which include one or both products of $\delta$ 's are of the same structure as considered earlier. They are

$$
1-(1+i a T(b))^{A}-\left(1-i a^{*} T(b)\right)^{A} .
$$

Some care should be taken in calculating the remaining term coming from the product of the $2 A S$-matrices. As the wave function factorizes (Eq. (2)), the i-th term is

$$
\sum_{\alpha_{i}, \beta_{i}, \alpha_{i}^{\prime}} \chi_{\alpha_{i}}^{*} \chi_{\alpha_{i}^{\prime}} \int d^{2} b_{i} T\left(b_{i}\right)\left(\delta_{\alpha_{i} \beta_{i}}+i a_{\alpha_{i} \beta_{i}}\left(b-b_{i}\right)\right)\left(\delta_{\alpha_{i}^{\prime} \beta_{i}}-i a_{\alpha_{i}^{\prime} \beta_{i}}^{*}\left(b-b_{i}\right)\right) \text {. }
$$


Doing the integration and the sum over $\alpha$ 's, $\alpha^{\prime \prime}$ s and $\beta$ 's, we obtain

$$
\sigma_{e l+d(A)}^{h A}(b)=1+T(b)\left[i a-i a^{*}+\sum_{\alpha_{i}, \alpha_{i}^{\prime}} \chi_{\alpha_{i}}^{*} \chi_{\alpha_{i}^{\prime}} \int d^{2} b \sum_{\beta_{i}} a_{\alpha_{i} \beta_{i}} a_{\alpha_{i}^{\prime} \beta_{i}}^{*}\right]
$$

The integral term represents the cross section for the interaction of the projectile with a nucleon from the target with all its possible excitations included. Physically it is a cross section for events in which apart from the projectile only particles appear which have their rapidities smaller than $y_{\max }$. It depends on $y_{\max }$ and we denote it

$$
\sigma\left(y_{\max }\right)=\sum_{\alpha_{i}, \alpha_{i}^{\prime}} \chi_{\alpha_{i}}^{*} \chi_{\alpha_{i}^{\prime}} \int d^{2} b \sum_{\beta_{i}} a_{\alpha_{i} \beta_{i}} a_{\alpha_{i}^{\prime} \beta_{i}}^{*} .
$$

The unitarity condition gives

$$
i a-i a^{*}=-\sigma^{t o t},
$$

where the total cross section $\sigma^{\text {tot }}$ is defined by an expresion similar to Eq. (11) in which the summation is extended to all the existing states (not anly belonging to an excited nucleon as in this equation). Such a situation corresponds to choosing $y_{\max }$ equal to the projectile rapidity $Y$ (then all possible produced particles will be taken into account in Eq. (11)). So, we have

$$
\sigma(Y)=\sigma^{t o t}
$$

In the opposite limit $y_{\max } \rightarrow 0$, the cross section $\sigma\left(y_{\max }\right)$ goes over into $\sigma^{e l}$ :

$$
\sigma(0)=\sigma^{e l}
$$

Therefore, using Eqs. (8) to (12), Eq. (7) gets into

$$
\begin{aligned}
\sigma_{e l+d(A)}^{h A}(b) & =1-(1+i a T(b))^{A}-\left(1-i a^{*} T(b)\right)^{A} \\
& +\left(1-\left[\sigma^{t o t}-\sigma\left(y_{\max }\right)\right] T(b)\right)^{A} \\
& =\sigma_{\text {tot }}^{h A}-\left(1-\left(1-\left[\sigma^{t o t}-\sigma\left(y_{\max }\right)\right] T(b)\right)^{A}\right)
\end{aligned}
$$

and

$$
\sigma_{d(A)}^{h A}(b)=\left(1-\left[\sigma^{t o t}-\sigma\left(y_{\max }\right)\right] T(b)\right)^{A}-|1+i a T(b)|^{2 A} .
$$

For $A \gg 1$,

$$
\sigma_{d(A)}^{h A}(b)=\exp \left(-A T(b)\left[\sigma^{t o t}-\sigma\left(y_{\max }\right)\right]\right)-\exp \left(-A T(b) \sigma^{t o t}\right) .
$$

For small $y_{\max }, \sigma\left(y_{\max }\right)$ is of the order of $\sigma^{e l}, \sigma^{t o t}-\sigma\left(y_{\max }\right)$ is of the order of $\sigma^{i n+d}$ and the two terms of Eq. (17) are small and of the same order of magnitude. The resulting dependence of the cross section integrated over impact parameter is then $A^{1 / 3}$. If $y_{\max }$ is large then $\sigma\left(y_{\max }\right)$ is of the order of $\sigma^{\text {tot }}$ and then the resulting dependence of Eq. (17) is $A^{2 / 3}$. Eq. (16) or (17) describes a smooth transition between the two regimes as the threshold rapidity moves from the target to the projectile fragmentation region. 
To compute the projectile diffraction dissociation one has to assume that the incident hadron is a superposition of $N$ parton states $\left|\vec{b}_{1}, \ldots, \vec{b}_{N}, y_{1}, \ldots, y_{N}\right\rangle$ which are the eigenstates of diffraction on a nucleus with $A$ nucleons at impact parameters $\vec{b}_{1}^{\prime}, \ldots . \vec{b}_{A}^{\prime}$. The scattering amplitude is

$$
M\left(\vec{b}_{1}, \ldots, \vec{b}_{N}, y_{1}, \ldots y_{N} ; \vec{b}_{1}^{\prime}, \ldots, \vec{b}_{.4}^{\prime}\right)=1-\prod_{i=1}^{N} \prod_{j=1}^{A}\left[1-\tau\left(\vec{b}_{i}-\vec{b}_{j}^{\prime}+\vec{B}, y_{i}\right)\right],
$$

where $\vec{B}$ is the impact parameter of the incident hadron and $\vec{b}_{i}$ and $\vec{b}_{j}^{\prime}$ are measured from the center of the incident hadron and the nucleus respectively. $\tau$ is the hadron-nucleon amplitude. The projectile single diffractive cross section is ([15])

$$
\frac{d \sigma_{d(p)}}{d^{2} B}=\left\langle M^{2}\right\rangle-\langle M\rangle^{2}
$$

where the subscript $d(p)$ means diffractive dissociation of the projectile and the averages are taken over the probability distribution of the beam partons and also over the ground state distribution of nucleons in the target nucleus.

Finally the total diffractive cross section including beam, target and both beam and target dissociation can be obtained ([16]) from Eq. (6),

$$
\sigma_{i n+d}^{h A}(b) \simeq 1-\left(1-\sigma^{t o t} T(b)\right)^{A}=\sum_{n=1}^{A} C_{A}^{n}\left(\sigma^{t o t} T(b)\right)^{n}\left(1-\sigma^{t o t} T(b)\right)^{A-n} .
$$

As $\sigma^{t o t}=\sigma^{i n}+\sigma^{e l+d}$, we have $([17])$

$$
\sigma_{i n}^{h A}(b)=\sum_{n=1}^{A} C_{A}^{n}(T(b))^{n} \sum_{j=1}^{n} C_{n}^{j}\left(\sigma^{i n}\right)^{j}\left(\sigma^{e l+d}\right)^{n-j}\left(1-\sigma^{t o t} T(b)\right)^{A-n},
$$

so

$$
\sigma_{i n}^{h A}(b)=1-\left(1-\sigma^{i n} T(b)\right)^{A}
$$

Therefore

$$
\sigma_{d}^{h A}(b)=\sigma_{i n+d}^{h A}(b)-\sigma_{i n}^{h A}(b)=\left(1-\sigma^{i n} T(b)\right)^{A}-|1+i a T(b)|^{2 A}
$$

\section{The probabilistic model of fusion of strings in hadron-nucleus collisions}

The standard Glauber treatment of the $h A$ interaction is based on the assumption that collisions of the projectile with individual nucleons of the target nucleus are independent. For the scattering matrix at a given impact parameter $b$ one then gets

$$
S^{(A)}(b)=\int\left[\prod_{i=1}^{A} d^{2} b_{i}\right] T_{A}\left(b-b_{1}, \ldots, b-b_{A}\right)\left[\prod_{i=1}^{A} S^{(1)}\left(b_{i}\right)\right]
$$


where $S^{(1)}(b)$ is the $h N$ scattering matrix and $T_{A}$ is the probability density to find the $\mathrm{i}-\mathrm{th}$ nucleon at the transverse distance $b_{i}$ from the center. If nucleon correlations are neglected, $T_{A}$ factorizes,

$$
T_{A}\left(b-b_{1}, \ldots, b-b_{A}\right)=\prod_{i=1}^{A} T_{A}\left(b-b_{i}\right) .
$$

The probability picture of string interactions naturally leads to the interaction of strings produced in collisions of the projectile with different nucleons. This will destroy the factorizable structure and then the Glauber formula.

To introduce the interaction of strings originating from different nucleons ([6]) we have to take into account the spatial interpretation of Eq. (24). Putting inside the integral in this equation

$$
1=\theta\left(R_{0}-b_{i}\right)+\theta\left(b_{i}-R_{0}\right)
$$

for each integration, we split the whole transverse space for each nucleon into the interacion area, of size $s_{0}=\pi R_{0}^{2} \simeq \sigma^{i n}$ around the projectile, and the rest where there is no interaction. Consider the contribution with $l$ nucleons in the interaction area (that is, coming from $l$ functions $\left.\theta\left(R_{0}-b_{i}\right)\right)$. It contains a symmetry factor $C_{A}^{l}$. With $b_{i} \leq R_{0}$ we can neglect $b_{i}$ as compared with the overall impact parameter $b$, which contributes a factor $\left(T_{A}(b)\right)^{l}$. Finally, $S^{(1)}\left(b_{i}\right)=1$ for the nucleons with $b_{i} \geq R_{0}$, so that each corresponding integration in Eq. (24) will give a factor $\left(1-s_{0} T_{A}(b)\right)$. As a result, the total $h A$ scattering matrix will be given by

$$
S^{(A)}(b)=\sum_{l=0}^{A} S_{l}^{(A)}(b)
$$

where

$$
S_{l}^{(A)}(b)=C_{A}^{l}\left(T_{A}(b)\right)^{l}\left(1-s_{0} T_{A}(b)\right)^{A-l} \int_{s_{0}}\left[\prod_{i=1}^{l} d^{2} b_{i}\right] S_{l, b_{1}, \ldots, b_{l}}^{(A)},
$$

$S_{l, b_{1}, \ldots, b_{l}}^{(A)}$ being the scattering matrix with the position of the $l$ nucleons fixed in the interaction area (in the Glauber model is $\prod_{i=1}^{l} S^{(1)}\left(b_{i}\right)$ ).

To introduce the interaction of strings in $h N$ we do the following: The $S$-matrix can be written in the eikonal approximation as a sum of contributions coming from the exchange of $N$ Pomerons multiplied by their probability,

$$
S^{(1)}=\sum_{N} p_{N} s^{N}(b)=\exp (Q[s(b)-1])
$$

with

$$
p_{N}=\exp (-Q) \frac{Q^{N}}{N !}
$$

The fusion of strings is included by changing $p_{N}$ to

$$
p_{N}=c \frac{Q^{N}}{N !} \prod_{k=1}^{N-1}(1-k x)
$$


where $x$ is the probability of fusion of two Pomerons into one ([5]).

More general, one can have different type of Pomerons coming from the fusion of a different number of Pomerons. The Pomerons of "colour numbers" $n_{1}, n_{2}, \ldots$ fuse into one with colour number $n=\sum n_{i}$. Let be $\nu_{n}$ the number of Pomerons with colour number $n$. Then the total number of Pomerons is $M=\sum \nu_{n}$ and the total colour number is $N=\sum n \nu_{n}$. In this case

$$
S^{(1)}(b)=S(b)=\sum_{\nu_{n}} p\left(\nu_{n}\right) \prod\left(s_{n}(b)\right)^{\nu_{n}}
$$

with

$$
p\left(\nu_{n}\right)=c \frac{Q^{N}}{\prod \nu_{n} ! \prod(n !)^{\nu_{n}}} x^{N-M} \prod_{k=1}^{M-1}(1-k x) .
$$

Coming back to $h A$ collisions, it is natural to assume that not only strings coming from the same target nucleon may fuse, but also the ones coming from different nucleons which are all in the same interaction area. This leads to ([6])

$$
S_{l, b_{1}, \ldots, b_{l}}^{(A)}=\prod_{i=1}^{l}\left[\sum_{N_{i}}\left(s\left(b_{i}\right)\right)^{N_{i}}\right] p_{N_{1}, \ldots, N_{l}}^{l},
$$

with the probability

$$
p_{N_{1}, \ldots, N_{l}}^{l}=c_{l} \prod_{i=1}^{l} \frac{Q^{N_{i}}}{N_{i} !} \prod_{k=1}^{N-1}(1-k x),
$$

$c_{l}$ being the normalization constant $1 /\{1+l g\}^{1 / x}$ (we denote by $\{a+b\}^{\alpha}$ the part of $(a+b)^{\alpha}$ with positive binomial coefficients), $g=Q x$ and $N_{i}$ the number of strings formed with the $\mathrm{i}$-th nucleon of the target. The final result is

$$
S_{l, b_{1}, \ldots, b_{l}}^{(A)}=\frac{\left\{1+g s_{l}\left(b_{1}, \ldots, b_{l}\right)\right\}^{1 / x}}{\{1+l g\}^{1 / x}}
$$

with

$$
s_{l}\left(b_{1}, \ldots, b_{l}\right)=\sum_{i=1}^{l} s\left(b_{i}\right)
$$

The cross section $\sigma_{l k}^{(A)}\left(b_{1}, \ldots, b_{l}\right)$ which corresponds to $k$ cut Pomerons and an arbitrary number of uncut ones is easily deduced for Eq. (36) using the AGK cutting rules ([18]),

$$
\sigma_{l k}^{(A)}\left(b_{1}, \ldots, b_{l}\right)=\left(\sigma_{l}\left(b_{1}, \ldots, b_{l}\right)\right)^{k} C_{1 / x}^{k} \frac{\left\{1+l g-\sigma_{l}\left(b_{1}, \ldots, b_{l}\right)\right\}^{1 / x-k}}{\{1+l g\}^{1 / x}} .
$$

In the case of various types of Pomerons the formulae (34), (35), (36) and (37) should be replaced by:

$$
S_{l, b_{1}, \ldots, b_{l}}^{(A)}=\prod_{i=1}^{l}\left[\sum_{\nu_{n}^{(i)}}\left(s_{n}\left(b_{i}\right)\right)^{\nu_{n}^{(i)}}\right] p^{(l)}\left(\nu_{n}^{(i)}\right)
$$




$$
\begin{gathered}
p^{(l)}\left(\nu_{n}^{(i)}\right)=c_{l} \frac{Q^{N}}{\prod_{i, n}\left(\nu_{n}^{(i)}\right) !(n !)^{\nu_{n}^{(i)}}} x^{N-M} \prod_{k=1}^{M-1}(1-k x) \\
\left(N=\sum_{i, n} n \nu_{n}^{(i)}, \quad M=\sum_{i, n} \nu_{n}^{(i)}\right), \\
S_{l, b_{1}, \ldots, b_{l}}^{(A)}=\frac{\left\{1+s_{l}^{(r)}\left(b_{1}, \ldots, b_{l}\right)\right\}^{1 / x}}{\{1+l g\}^{1 / x}}
\end{gathered}
$$

and

$$
\begin{aligned}
s_{l}^{(r)}\left(b_{1}, \ldots, b_{l}\right) & =\sum_{i=1}^{l} s^{(r)}\left(b_{i}\right), \quad s^{(r)}(b)=\sum_{n=1}^{\infty} s_{n}(b) \frac{(Q x)^{n}}{n !}=g+i a^{(r)}(b) \\
g & =\exp (Q x)-1, \quad a^{(r)}(b)=\sum_{n=1}^{\infty} a_{n}(b) \frac{(Q x)^{n}}{n !} .
\end{aligned}
$$

The cross section corresponding to $k_{n}$ cut Pomerons of type $n$ for fixed $l, b_{1}, \ldots, b_{l}$ is

$$
\begin{aligned}
& \sigma_{l, k_{1}, \ldots, k_{l}}^{(A)}\left(b_{1}, \ldots, b_{l}\right)=\frac{k !}{\prod_{n} k_{n} !}\left[\prod_{n} z_{n}^{k_{n}}\right] \sigma_{l k}^{(A)} \\
& k=\sum_{n} k_{n}, \quad z_{n}=\frac{\sigma_{n}^{(r)}}{\sigma_{0}} \text { and } \sum_{n} z_{n}=1 .
\end{aligned}
$$

Once we know the $S$-matrix, the total $\sigma_{\text {tot }}^{h A}$, elastic $\sigma_{e l}^{h A}$ and total inelastic $\sigma_{i n+d}^{h A}$ cross sections are computed in the usual way:

$$
\begin{gathered}
\sigma_{\text {tot }}^{h A}(b)=2-S^{(A)}(b)-\left(S^{(A)}(b)\right)^{*} \\
\sigma_{e l}^{h A}(b)=\left|1-S^{(A)}(b)\right|^{2} \text { and } \\
\sigma_{i n+d}^{h A}(b)=1-\left|S^{(A)}(b)\right|^{2} .
\end{gathered}
$$

The inelastic non-diffractive cross section $\sigma_{i n}^{h A}$ is obtained in the following way: From Eq. (38) we get

$$
\sigma_{l k}^{(A) i n}\left(b_{1}, \ldots b_{l}\right)=\left(\sigma_{l}^{i n}\left(b_{1}, \ldots, b_{l}\right)\right)^{k} C_{1 / x}^{k} \frac{\left\{1+l g-\sigma_{l}^{i n}\left(b_{1}, \ldots, b_{l}\right)\right\}^{1 / x-k}}{\{1+l g\}^{1 / x}}
$$

where

$$
\sigma_{l}^{i n}\left(b_{1}, \ldots, b_{l}\right)=\sum_{i=1}^{l} \sigma_{0}^{i n}\left(b_{i}\right), \quad \Omega^{0} \equiv \frac{\sigma_{0}^{i n}}{\sigma_{0}^{i n+d}}
$$

$\sigma_{0}^{i n}\left(b_{i}\right)$ being the elementary inelastic non-diffractive cross section. Once $\sigma_{l k}^{(A) \text { in }}$ is computed, the inelastic non-diffractive cross section $\sigma_{i n}^{h A}$ is obtained integrating over all $b_{i}$ 's in the interaction area, then summing over $l=1, \ldots, A$ with the weights $C_{A}^{l}\left(T_{A}(b)\right)^{l}(1-$ $\left.s_{0} T_{A}(b)\right)^{A-l}$ and finally integrating over the overall impact parameter $b$.

In the case of Pomerons of various types, we proceed in a similar way beginning with Eq. (42) instead of Eq. (36).

In both cases, the inelastic diffractive cross section $\sigma_{d}^{h A}$ is obtained substracting the inelastic non-diffractive from the total inelastic cross section,

$$
\sigma_{d}^{h A}=\sigma_{i n+d}^{h A}-\sigma_{i n}^{h A}
$$




\section{Comparison of Glauber and string fusion models}

First of all let us consider the hadron-hadron case ([9]) where we can write the $S$-matrix in the string fusion model in the general case of Pomerons of different type as

$$
S(b)=\left(1+i \frac{\tilde{a}(b)}{1+g}\right)^{1 / x}
$$

(we consider, for simplicity, $1 / x$ being an integer and the amplitude $\tilde{a}$ purely imaginary), where $\tilde{a}(b)=a^{(r)}(b)$ and $g$ are given in Eq. (44).

There are two extreme cases:

a) $x \rightarrow 0$ and $Q x \rightarrow 0$. In this case $g \rightarrow 0, \tilde{a} /(1+g) \rightarrow a_{1} Q x \rightarrow 0$ and

$$
S(b) \simeq \exp \left(i a_{1}(b) Q\right)
$$

which is just the eikonal case. being $i a_{1} Q$ the contribution of one Pomeron. This case corresponds to no interaction of strings.

b) $x \rightarrow 0$ and $Q x \gg 1$, but $\tilde{a}<g$. Then

$$
S(b) \simeq \exp \left[i \frac{\tilde{a}(b)}{(1+g) x}\right]
$$

which again takes an eikonal form but now with an effective Pomeron $i \tilde{a} /[(1+g) x]$.

In hadron-nucleus collisions, from Eqs. (42), (43) and (44) we have

$$
S_{l . b_{1}, \ldots, b_{l}}^{(A)}=\left(1+i \frac{\sum_{i=1}^{l} \tilde{a}\left(b_{i}\right)}{1+l g}\right)^{1 / x} .
$$

If $l \gg 1$, then in both cases a) and b) $i \sum \tilde{a}\left(b_{i}\right) /(1+l g) \rightarrow 0$ and

$$
S_{l, b_{1}, \ldots, b_{l}}^{(A)} \simeq \exp \left\{i \frac{\sum_{i=1}^{l} \tilde{a}\left(b_{i}\right)}{x(1+\lg )}\right\},
$$

which can be integrated over each $b_{i}$

$$
\int d^{2} b_{i} \exp \left\{i \frac{\tilde{a}\left(b_{i}\right)}{x(1+l g)}\right\}=s_{0}+i \alpha_{l}
$$

where

$$
i \alpha_{l}=\int d^{2} b_{i}\left(\exp \left\{i \frac{\tilde{a}\left(b_{i}\right)}{x(1+l g)}\right\}-1\right)
$$

Therefore

$$
\int\left[\prod_{i=1}^{l} d^{2} b_{i}\right] S_{l, b_{1}, \ldots, b_{l}}^{(\mathcal{A})} \simeq\left(s_{0}+i \alpha_{l}\right)^{l}
$$


and

$$
S^{(-A)}(b) \simeq \sum_{l=0}^{A} C_{A}^{l}\left(T_{A}(b)\right)^{l}\left(1-s_{0} T_{A}(b)\right)^{A-l}\left(s_{0}+i \alpha_{l}\right)^{l} .
$$

In the case a) (no interaction of strings) $g \rightarrow 0$ and $\alpha_{l}$ does not depend on $l$, then

$$
S^{(A)}(b) \simeq\left(1-s_{0} T_{A}(b)+\left(s_{0}+i \alpha\right) T_{A}(b)\right)^{A}=\left(1+i \alpha T_{A}(b)\right)^{A},
$$

that is, the Glauber model reobtained.

In the case b) $(Q x \gg 1)$,

$$
i \alpha_{l} \simeq \frac{1}{x l g} \int d^{2} b i \tilde{a}(b)+\frac{1}{2(x l g)^{2}} \int d^{2} b(i \tilde{a}(b))^{2}+\cdots
$$

and

$$
\begin{aligned}
\left(s_{0}+i \alpha_{l}\right)^{l} & =s_{0}^{l}\left(1+i \alpha_{l} / s_{0}\right)^{l} \simeq s_{0}^{l} \exp \left\{l\left[i \frac{\alpha_{l}}{s_{0}}-\frac{1}{2}\left(i \frac{\alpha_{l}}{s_{0}}\right)^{2}+\cdots\right]\right\} \\
& \simeq s_{0}^{l} \exp \left(i \int d^{2} b \frac{\tilde{a}(b)}{g s_{0} x}\right) \simeq s_{0}^{l} \exp \left(i \int d^{2} b \frac{p(b)}{s_{0}}\right) \\
& =s_{0}^{l} \exp \left(i \frac{\tilde{p}(0)}{s_{0}}\right)
\end{aligned}
$$

where

$$
i p(b) \equiv i \frac{\tilde{a}(b)}{(1+g) x}
$$

is the effective Pomeron and $\tilde{p}(0)$ its expression in the space of transverse momentum. Therefore

$$
S^{(A)}(b) \simeq \exp \left(i \frac{\tilde{p}(0)}{s_{0}}\right) .
$$

We assume that $T_{A}(b)=\theta\left(R_{A}-b\right) /\left(\pi R_{A}^{2}\right)$. This expresion is valid for $T_{A}(b)>0$; for $T_{A}(b)=0, S^{(A)}(b)=1$. Eq. $(65)$ does not depend on $A$ but is not the same as $S^{(1)}(b)=$ $\exp [i p(b)]$.

The diffractive cross section will be

$$
\sigma_{d}^{h A}(b)=\theta\left(R_{A}-b\right)\left[\exp \left(-\sigma_{P}^{i n} / s_{0}\right)-\exp \left(-\sigma_{P}^{t o t} / s_{0}\right)\right]
$$

where

$$
\sigma_{P}^{t o t}=2 \operatorname{Im} \tilde{p}(0), \quad \sigma_{P}^{e l}=\int d^{2} b(p(b))^{2}, \quad \sigma_{P}^{i n}=\Omega^{0}\left(\sigma_{P}^{t o t}-\sigma_{P}^{e l}\right),
$$

to compare with the Glauber formula (23),

$$
\sigma_{d}^{h A}(b)=\theta\left(R_{A}-b\right)\left[\exp \left(-A T_{A}(b) \sigma^{i n}\right)-\exp \left(-A T_{A}(b) \sigma^{t o t}\right)\right],
$$

where now

$$
\sigma^{t o t}=2 \operatorname{Im} \int d^{2} b[1-\exp (i p(b))], \quad \sigma^{e l}=\int d^{2} b|1-\exp (i p(b))|^{2} .
$$


First, notice that Eq. (65) coincides with the Glauber $S$-matrix

$$
S^{(A)}(b)=\left(1+i a^{e f f} T_{A}(b)\right)^{A_{e f f}} \simeq \exp \left(i a^{e f f} T_{A}(b) A_{e f f}\right),
$$

corresponding to a number of nucleons $A_{\text {eff }}$ and an effective nucleon-nucleon amplitude $a^{e f f}$ if

$$
\frac{\tilde{p}(0)}{s_{0}}=A_{e f f} \frac{a^{e f f}}{\pi R_{A}^{2}}
$$

which is satisfied if

$$
a^{e f f}=\tilde{p}(0) \text { and } A_{e f f}=\frac{\pi R_{0}^{2}}{s_{0}} A^{2 / 3} \sim A^{2 / 3} .
$$

Eq. (72) means that, in this approximation of strong fusion, the $S$-matrix coincides with the $S$-matrix given by the Glauber model taking as the nucleon-nucleon amplitude the effective Pomeron and the number of nucleons $A^{2 / 3}$ instead of $A$, although the profile function remains equal, $T_{A}(b)$.

Coming back to diffraction, Eqs. (66) and (68) can be approximately written as

$$
\sigma_{d}^{h A}(b)=\theta\left(R_{A}-b\right)\left[\exp \left(-\sigma^{i n} / s_{0}\right)-\exp \left(-\sigma^{t o t} / s_{0}\right)\right]
$$

and

$$
\sigma_{d}^{h A}(b)=\theta\left(R_{A}-b\right)\left[\exp \left(-A^{1 / 3} \sigma^{i n} / s_{0}\right)-\exp \left(-A^{1 / 3} \sigma^{t o t} / s_{0}\right)\right] .
$$

showing that the string fusion diffractive cross section is larger than the Glauber one. The first one goes like $A^{2 / 3}$ and the second one like $A^{1 / 3}$.

\section{Results and comparison with other models and experimental data}

Following the above sections we compute the diffractive cross section in the case of Pomerons of the same type and of different types. We use the following parametrization of the elementary amplitude:

$$
\begin{aligned}
& \sigma(b)=\sigma(0) \exp \left(-b^{2} / R^{2}\right), \quad \sigma(0)=c\left(2 R_{0} / R\right)^{2} s^{\Delta}, \\
& R^{2}=4\left(R_{0}^{2}+\alpha^{\prime} \ln s\right), \quad \Delta=0.145, \quad \alpha^{\prime}=0.25 \mathrm{GeV}^{-2}, \\
& R_{0}^{2}=3.56 \mathrm{GeV}^{-2}, \quad c=0.40, \quad s_{0}=\sigma_{h N}^{i n}=26.2 \mathrm{mb}, \quad x=0.05
\end{aligned}
$$

and Gaussians for the profile functions of the nuclei. In Table 1 the results for the diffractive cross sections are shown for different nuclei at $\sqrt{s_{N N}}=19.4 \mathrm{AGeV}$. The values of the parameters are chosen to reproduce the $N N$ cross sections and $\sigma_{i n+d}^{h A}$ in the Glauber model.

From this table it is seen that the diffractive cross section does not depend on including different types of Pomerons or the same type. The bulk of the diffractive cross section 
is already obtained when $\Omega^{0}=1$, that is, when there is no diffraction in the elementary amplitude. This differs from the situation in hadron-hadron collisions but is expected due to the existence of many excited nucleonic states in the nucleus. Lowering the value of $\Omega^{0}$ from 1 to 0.7 , that is, introducing some diffraction in the elementary amplitude, the diffractive cross section rises around 20 to 25 per cent. The obtained final $\Omega_{f}^{0}=\sigma_{i n}^{h A} / \sigma_{i n+d}^{h A}$ is smaller than the $\Omega^{0}$ input. For instance, for $A=12$ and $\Omega^{0}$ in the range 0.7 to 1 , the $\Omega_{f}^{0}$ obtained varies from 0.65 to 0.72 .

The $A$-dependence of the diffractive cross section lies between $A^{2 / 3}$ and $A^{1 / 3}$ as it was expected and the $b$ dependence is shown in Fig. 2 for $A=7$ for different $\Omega^{0}$ 's. It is mostly peripheral (around the radius of the nucleus) although there are non negligible contributions from central impact parameters. These contributions are responsible for the departure from the usual $A^{1 / 3}$ behaviour.

The results for the Glauber model are presented in Table 2, using Gaussian profile functions for $A<16$ and Wood-Saxon for $A \geq 16$. The input values used are:

$$
\sigma_{h N}^{i n}=26.2 \mathrm{mb}, \quad \sigma_{h N}^{\text {tot }}=38 \mathrm{mb} \text { and } \sigma_{h N}^{e l}=\sigma\left(y_{\max }\right)=5.6 \mathrm{mb} .
$$

Experimental data on diffraction in hadron -nucleus collisions are rather scarce. Refering to diffraction of the beam, the existing data are concentrated in specific channels and there are no data on the total diffraction of the beam. Regarding the diffraction of the nucleus we have found only the recent data of the HELIOS Collaboration ([14]). In Table 3 we present these results together with the predictions in the Glauber model, in a DPM Monte Carlo ([13]) and in the Faessler model ([19]). Also, the diffraction of the projectile of Ref. [15] and in the Faessier model and the total diffractive cross section in the Glauber model and in the string fusion model are presented.

The range of values quoted for $\sigma_{d(A)}^{h A}$ and $\sigma_{d(p)}^{h A}$ in the Faessler model corresponds to different values of the parameters of the model and even in the most favourable case the values of $\sigma_{d(A)}^{h A}$ do not agree with the experimental data. They lie above, as well as the DPM and Glauber predictions do. $\sigma_{d(p)}^{h A}$ in the Faessler model and in Ref. [15] are obtained by the same method and formula and this is the reason for their similar results. The variation in the values of $\sigma_{d(A)}^{h A}$ in the DPM for fixed $A$ is due to differences which appear regarding the possibility of fluctuations in the nucleon-nucleon cross sections. The single diffractive hadron-nucleus interaction is introduced in the Monte Carlo code of the DPM as a Glauber process involving only one single target nucleon and assuming that a fraction $\sigma_{s d}^{h N} / \sigma_{i n+d}^{h N}$ of the events are diffractive. These values are obtained from a fit to the nucleon-nucleon diffractive cross section using triple Pomeron couplings to leading order. The values quoted correspond to similar kinematical cuts to the experimental data. This can be the reason for obtaining smaller values than the Glauber ones. Anyway, the values are larger than the experimental data.

The values obtained with string fusion are larger than the Glauber ones. We have computed only up to $A=12$ due to the time needed to compute the $b_{i}$ integrals. On the other hand, the trend of $\sigma_{d}^{h A}$ is already seen. The detailed comparison at not very high energy could be affected by finite energy effects. In fact at $\sqrt{s}=20 \mathrm{GeV}$ the exchange of a very few strings is expected (for instance for $A=12$ no more than 3 ) and therefore the 
effects of string fusion are quite small. These effects should appear only at much higher energies in $h A$ collisions. Finite energy limitations have not been taken into account in our formulae. To compute the exact values of diffraction a detailed Monte Carlo simulation of the string fusion model should be necessary as realized in Refs. [7] and [8]. Therefore our values should be regarded as showing the general trend.

\section{Conclusions}

The diffractive cross section for hadron-nucleus collisions has been computed in the string fusion model and compared with different models, specially with the Glauber model. The existing scarce experimental data prevent us from doing a detailed comparison. Our main conclusion is that the effect of string fusion concerning the diffractive events in hadron-nucleus collisions is to increase the cross section in comparison with the Glauber model. This could have interesting consecuences in the Higgs hunting. One of the proposed ways to detect the Higgs particle is through the processes $p p \rightarrow j e t+H+j e t$ where the particles of each jet are produced with rapidities very close to the rapidities of each incoming proton respectively ([20], [21]). Therefore, between the particles of each jet and the particles coming from the decay of the Higgs particle there will be a large rapidity gap. Then, the so-called "survival probability" ([21], [22], [23]), which is the probability for this gap not to be filled with particles. is crucial. It is strongly related to the diffractive cross section. In hadron-nucleus collisions both the Higgs particle and the diffractive cross section are enhanced. Therefore the signature proposed is enhanced too. The effect of string fusion will then enhance it still more.

\section{Acknowledgements}

We thank N. S. Amelin for usefull discussions and the CICYT and the DGPCT of Spain and the Xunta de Galicia for financial support. 


\section{References}

[1] A. Capella, U. P. Sukhatme, C.-I. Tan and J. Tran Thanh Van, Phys. Lett. B81 (1979) 68; Phys. Rep. 236 (1994) 225.

[2] A. B. Kaidalov and K. A. Ter-Martirosyan, Phys. Lett. B117 (1982) 247.

[3] B. Andersson, G. Gustafson and B. Nilsson-Almqvist, Nucl. Phys. B281 (1987) 289.

[4] B. Andersson. Proceedings of the XXII International Symposium on Multiparticle Dynamics, Santiago de Compostela, July 1992 , Ed. C. Pajares, World Scientific 1993, p. 428.

[5] M. A. Braun and C. Pajares, Nucl. Phys. B390 (1993) 542.

[6] M. A. Braun and C. Pajares, Phys. Lett. B287 (1992) 154; Nucl. Phys. B390 (1993) 559 .

[7] N. S. Amelin, M. A. Braun and C. Pajares, Phys. Lett. B306 (1993) 312; Santiago preprint US-FT/92-18 (submitted to Z. Phys. C).

[8] C. Merino, C. Pajares and J. Ranft, Phys. Lett. B276 (1992) 168; H.-J. Möhring, J. Ranft, C. Merino and C. Pajares, Phys. Rev. D47 (1993) 4142.

[9] N. Armesto, M. A. Braun and C. Pajares, Phys. Rev. D48 (1993) 162.

[10] S. Barshay, P. Heiliger and D. Rein, Mod. Phys. Lett. A28 (1992) 2559.

[11] R. J. Glauber, Proceedings of the $2^{\text {nd }}$ International Conference on High Energy Physics and Nuclear Structure, Rehovot, 1967, Ed. G. Alexander, North Holland 1967.

[12] V. M. Braun and Yu. M. Shabelskii, Int. J. Mod. Phys. A11 (1988) 2417.

[13] J. Ranft and S. Roesler, CERN preprint CERN/TIS-RP/93-15/PP (submitted to Z. Phys. C).

[14] T. Åkesson et al., Z. Phys. C49 (1991) 355.

[15] H. Miettinen and J. Pumplin, Phys. Rev. Lett. 42 (1979) 204.

[16] C. Pajares and A. V. Ramallo, Il Nuovo Cimento A83 (1984) 1.

[17] R. Blankenbecler, A. Capella, C. Pajares, A. V. Ramallo and J. Tran Thanh Van, Phys. Lett. B107 (1981) 106.

[18] V. A. Abramovskii, V. N. Gribov and O. V. Kancheli, Yad. Fiz. 18 (1973) 595 (Sov. J. Nucl. Phys. 18 (1974) 308). 
[19] M. A. Faessler, Z. Phys. C58 (1993) 567.

[20] Y. L. Dokshitzer, V. Khoze and T. Sjöstrand, Phys. Lett. B274 (1992) 116.

[21] J. D. Bjorken, Phys. Rev. D47 (1993) 101.

[22] E. Gotsman, E. M. Levin and U. Maor, Phys. Lett. B309 (1993) 199.

[23] R. S. Fletcher, Phys. Lett. B320 (1994) 373. 


\section{Table captions}

Table 1. Hadron-nucleus cross sections for different nuclei $A$ at $\sqrt{s_{N N}}=19.4 \mathrm{AGeV}$ in the string fusion model: total $\left(\sigma_{t o t}\right)$, elastic $\left(\sigma_{e l}\right)$, total inelastic $\left(\sigma_{i n+d}\right)$ and inelastic diffractive in the case of Pomerons of the same type $\left(\sigma_{d}^{1}\right)$ and in the case of Pomerons of the different types with $a_{n}=a_{1}^{n}\left(\sigma_{d}^{2}\right)$, together with the Glauber total inelastic cross section $\left(\sigma_{i n+d}^{\text {Glau }}\right)$. The different values of the diffractive cross sections correspond to the different input values $\Omega^{0}$ shown. All the cross sections are in $m b$.

Table 2. Hadron-nucleus cross sections for different nuclei $A$ at $\sqrt{s_{N N}}=19.4 \mathrm{AGeV}$ in the Glauber model: total $\left(\sigma_{t o t}\right)$, total inelastic $\left(\sigma_{i n+d}\right)$, inelastic non-diffractive $\left(\sigma_{i n}\right)$, single inelastic diffractive of the nucleus using Eq. (16) $\left(\sigma_{d(A)}\right)$, single inelastic diffractive of the projectile taken from Ref. [15] $\left(\sigma_{d(p)}\right)$ and inelastic diffractive using Eq. (23) $\left(\sigma_{d}^{h A}\right)$. All the cross sections are in $m b$.

Table 3. Hadron-nucleus inelastic diffractive cross sections for different nuclei $A$ at $\sqrt{s_{N N}}=19.4 A G e V$ : experimental data $([14])$ on the diffraction of the nucleus $\left(\sigma_{d(A)}^{e x p}\right)$, Glauber results on the diffraction of the nucleus using Eq. (16) $\left(\sigma_{d(A)}^{\text {Glau }}\right)$, DPM Monte Carlo results ([13]) on the diffraction of the nucleus $\left(\sigma_{d(A)}^{D P M}\right)$, Faessler results ([19]) on the diffraction of the nucleus $\left(\sigma_{d(A)}^{F}\right)$, Glauber results $([15])$ on the diffraction of the projectile $\left(\sigma_{d(p)}^{\text {Glau }}\right)$, Faessler results $([19])$ on the diffraction of the projectile $\left(\sigma_{d(p)}^{F}\right)$, total $h A$ diffractive Glauber results using Eq. (23) $\left(\sigma_{d}^{\text {Glau }}\right)$ and total $h A$ diffractive string fusion results $\left(\sigma_{d}^{S F}\right)$. All the cross sections are in $m b$. 


\section{Figure captions}

Figure 1. Glauber picture of a $h A$ collision showing the lab rapidities: $y_{\max }$ which defines the diffractive states of the nucleus and $Y$ of the projectile.

Figure 2. $b$ dependence of the diffractive cross section $\sigma_{d i f f}(b)$ in the string fusion model for $A=7$, in the case of Pomerons of different types with $a_{n}=a_{1}^{n}$. The solid line corresponds to $\Omega^{0}=0.70$, the dashed one to $\Omega^{0}=0.82$ and the dotted one to $\Omega^{0}=0.95$. 
Table 1

\begin{tabular}{|c|c|c|c|c|c|c|c|}
\hline$A$ & $\Omega^{0}$ & $\sigma_{d}^{1}$ & $\overline{\sigma_{d}^{2}}$ & $\sigma_{t o t}$ & $\sigma_{e l}$ & $\sigma_{i n+d}$ & $\sigma_{\text {in }+d}^{\text {Glau }}$ \\
\hline \multirow{3}{*}{2} & 0.7 & 23.2 & 23.3 & \multirow{3}{*}{70.9} & \multirow{3}{*}{9.2} & \multirow{3}{*}{61.7} & \multirow{3}{*}{61.4} \\
\hline & 0.85 & 20.7 & 20.8 & & & & \\
\hline & 1 & 18.9 & 18.9 & & & & \\
\hline \multirow{3}{*}{4} & 0.7 & 41.4 & 41.6 & \multirow{3}{*}{132.8} & \multirow{3}{*}{19.4} & \multirow{3}{*}{113.4} & \multirow{3}{*}{113.1} \\
\hline & 0.85 & 37.0 & 37.1 & & & & \\
\hline & 1 & 33.8 & 34.0 & & & & \\
\hline \multirow{3}{*}{9} & 0.7 & 80.5 & 80.8 & \multirow{3}{*}{278.6} & \multirow{3}{*}{47.6} & \multirow{3}{*}{231.0} & \multirow{3}{*}{231.0} \\
\hline & 0.85 & 71.8 & 72.1 & & & & \\
\hline & 1 & 65.6 & 65.8 & & & & \\
\hline \multirow{3}{*}{12} & 0.7 & 101.4 & 101.8 & \multirow{3}{*}{362.2} & \multirow{3}{*}{65.4} & \multirow{3}{*}{296.8} & \multirow{3}{*}{297.0} \\
\hline & 0.85 & 90.3 & 90.7 & & & & \\
\hline & 1 & 82.5 & 82.8 & & & & \\
\hline
\end{tabular}

Table 2

\begin{tabular}{|c|c|c|c|c|c|c|}
\hline$A$ & $\sigma_{\text {tot }}$ & $\sigma_{\text {in }+d}$ & $\sigma_{i n}$ & $\sigma_{d(A)}$ & $\sigma_{d(p)}$ & $\sigma_{d}^{\text {hA }}$ \\
\hline 2 & 70.4 & 61.2 & 47.1 & 4.6 & & 14.2 \\
\hline 4 & 132.6 & 113.1 & 86.9 & 10.1 & & 26.2 \\
\hline 9 & 279.4 & 231.0 & 179.6 & 21.3 & & 51.4 \\
\hline 12 & 363.7 & 297.0 & 232.2 & 27.3 & 14 & 64.8 \\
\hline 27 & 666.3 & 480.6 & 402.1 & 32.2 & 25 & 78.4 \\
\hline 64 & 1286.5 & 847.8 & 742.8 & 43.0 & 46 & 105.0 \\
\hline 118 & 2011.6 & 1253.0 & 1128.7 & 50.7 & 64 & 124.3 \\
\hline 207 & 2997.7 & 1786.5 & 1643.5 & 58.5 & 90 & 143.0 \\
\hline
\end{tabular}


Table 3

\begin{tabular}{|c|c|c|c|c|c|c|c|c|}
\hline$A$ & $\sigma_{d(A)}^{\text {exp }}$ & $\sigma_{d(A)}^{\text {Glau }}$ & $\sigma_{d(A)}^{D P M}$ & $\sigma_{d(A)}^{F}$ & $\sigma_{d(p)}^{\text {Glau }}$ & $\sigma_{d(p)}^{F}$ & $\sigma_{d}^{\text {Glau }}$ & $\sigma_{d}^{S F}$ \\
\hline 2 & & 4.6 & & & & & 14.2 & 18.9 \\
\hline 4 & & 10.1 & & & & & 26.2 & 33.8 \\
\hline 9 & $8.4 \pm 1.5$ & 21.3 & & & & 10 & 51.4 & 65.6 \\
\hline 12 & & 27.3 & $17.0 \pm 1.1$ & & 14 & & 64.8 & 82.5 \\
\hline 27 & $13.3 \pm 2.6$ & 32.2 & $20.5 \pm 1.0$ & 21 & 25 & 23 & 78.4 & \\
\hline 64 & & 43.0 & $25.5 \pm 1.9$ & & 46 & & 105.0 & \\
\hline 118 & & 50.7 & & & 64 & & 124.3 & \\
\hline 184 & $23.5 \pm 4.5$ & & $31.2 \pm 2.8$ & $34-49$ & & $68-102$ & & \\
\hline 207 & & 58.5 & & & 90 & & 143.0 & \\
\hline
\end{tabular}




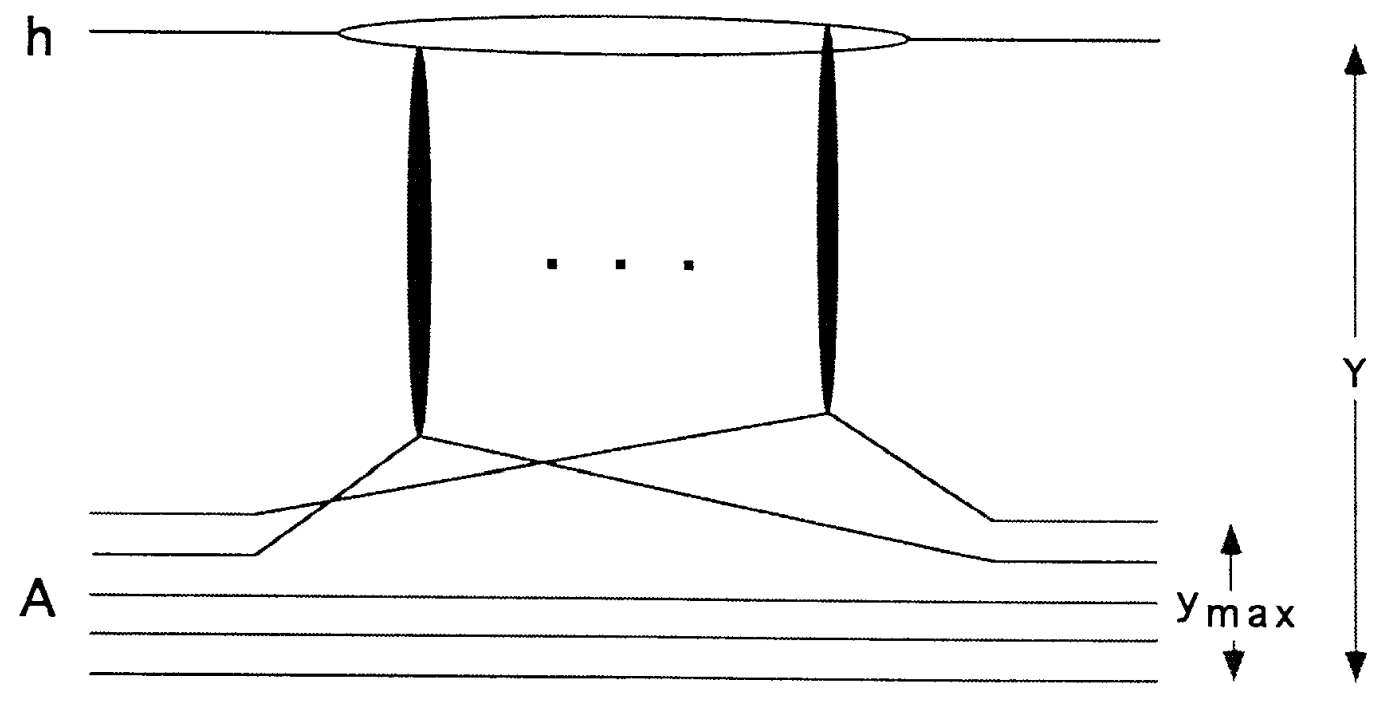

Fig. 1 
-.... 


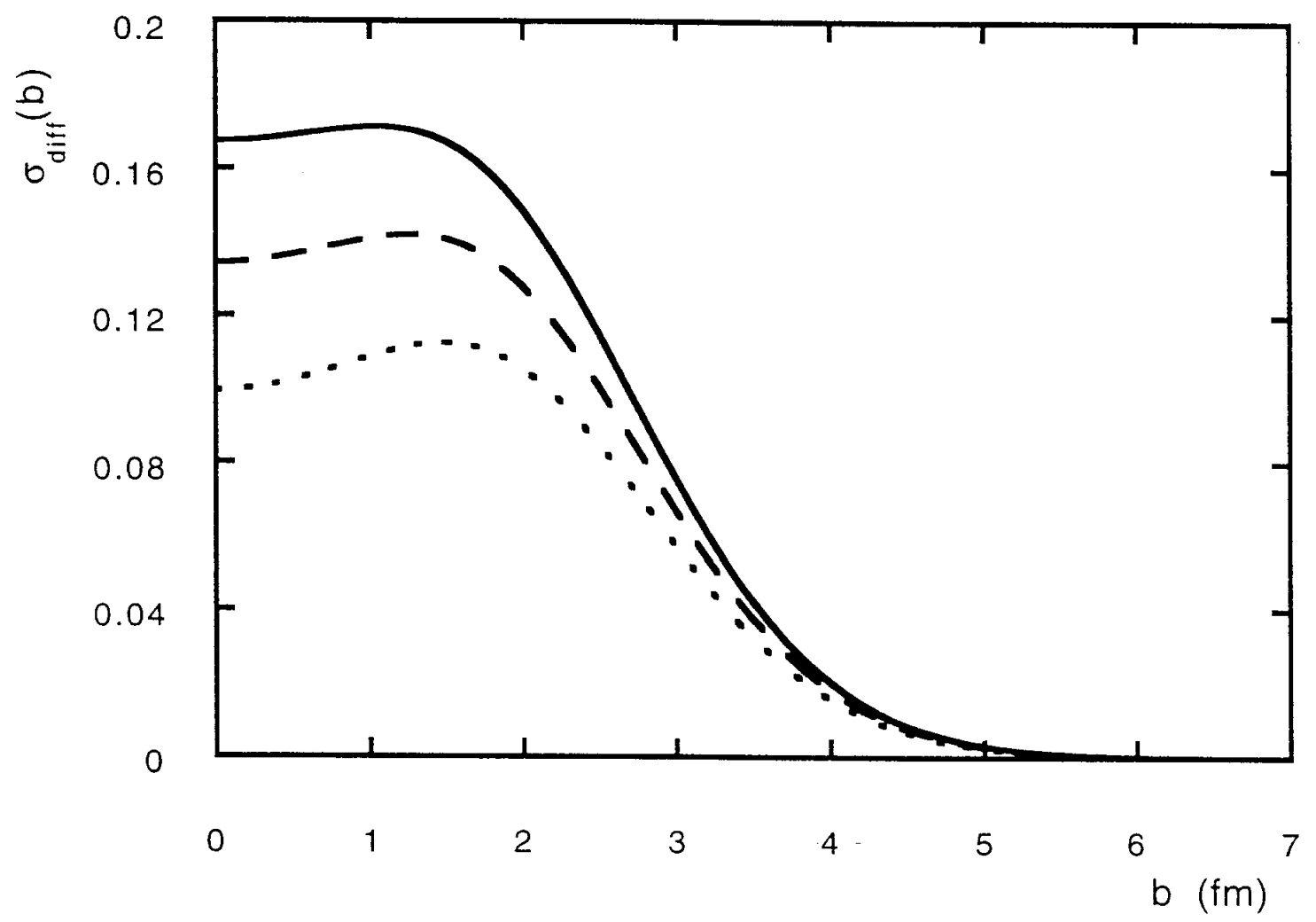

Fig. 2 
